\title{
False-positive result
}

National Cancer Institute ( $\mathrm{NCl})$

\section{Source}

National Cancer Institute (NCI). False-positive result.

A test result that indicates an individual is affected and/or has a certain gene mutation when he or she is actually unaffected and/or does not have the mutation; i.e., a positive test result in a truly unaffected or mutation-negative individual. 